\title{
DEPRESSÃO E ANSIEDADE NA ENFERMAGEM EM UNIDADE DE TERAPIA INTENSIVA
}

Depression and anxiety in nursing in an intensive care unit

Delecion y anxiety en la enfermedad en una unidad de cuidado intensivo

Malom Bhenson Tavares Barbosa • Discente do curso de Enfermagem do Centro Universitário Tabosa de Almeida - Associação Caruaruense de Ensino Superior (ASCES-UNITA) • E-mail: malombhenson@gmail.com

Daniela Barbosa de Lima Nascimento • Discente do curso de Enfermagem da ASCES-UNITA • E-mail: 2017106137@app.asces.edu.br

Rebeca Larissa Nepomuceno Torres • Discente do curso de Enfermagem da ASCESUNITA • E-mail: 2017106138@app.asces.edu.br

Carla Patrícia Pereira de Moraes • Discente do curso de Enfermagem da ASCESUNITA • E-mail: 2013106076@app.asces.edu.br

Erika Cristiane Soares da Silva • Discente do curso de Enfermagem da ASCESUNITA • E-mail: 2013206015@app.asces.edu.br

Manayra Windysa de Sousa Silva • Discente do curso de Enfermagem da ASCESUNITA • E-mail: 2013106069@app.asces.edu.br

Michel Gomes de Melo • Mestre e Docente de Curso de Enfermagem da ASCESUNITA • E-mail: michelmelo@asces.edu.br

Autor responsável pela correspondência:

Michel Gomes de Melo • E-mail: michelmelo@asces.edu.br 


\section{ciêncíncia
pural}

\section{RESUMO}

Introdução: A depressão é definida como uma síndrome, composta de diversos sintomas físicos e emocionais, com implicações sobre as capacidades comportamentais dos indivíduos. Numa Unidade de Terapia Intensiva, é quesito que o profissional possua agilidade na tomada de decisões, um cuidado livre de danos, conhecimento clínico e controle emocional. Esses elementos, agregados aos hábitos de vida, conflitos e estresse no ambiente laboral podem desencadear nesses profissionais transtornos como depressão e ansiedade. Objetivo: Identificar a prevalência de depressão e ansiedade em profissionais de enfermagem que atuam em Unidade de terapia intensiva adulto. Metodologia: Pesquisa descritiva, transversal com abordagem quantitativa. Por tratar-se de um censo, a população foi composta por toda equipe de enfermagem que atua em nessa unidade adulto de dois hospitais de grande porte da rede pública de saúde do município de Caruaru, estado de Pernambuco e que atendiam aos critérios de elegibilidade. Para a coleta de dados utilizou-se um questionário sociodemográfico e os inventários de depressão e ansiedade de Beck. Adotou-se estatística descritiva para a análise dos dados. Resultados: A média de idade dos profissionais foi de 35 anos, onde $86 \%$ são do gênero feminino e sua maioria são casados (46\%). Observou-se que $77 \%$ da amostra não apresenta sintomas depressivos. Já no que se refere aos sintomas ansiosos, $85 \%$ apresentaram grau mínimo de ansiedade. Conclusões: Os resultados desse estudo evidenciaram uma baixa prevalência de depressão e ansiedade. Tais resultados trazem aos gestores de saúde a possibilidade de atentar-se e rever as práticas adotadas nas instituições hospitalares. Recomenda-se que outros estudos, com análises mais robustas, sejam realizados no intuito de se ampliar o conhecimento sobre a temática.

Palavras-Chave: Depressão; Ansiedade; Saúde mental; Equipe de enfermagem; Unidades de Terapia Intensiva.

\section{ABSTRACT}

Introduction: Depression is defined as a syndrome, composed of several physical and emotional symptoms, with implications for the behavioral capacities of individuals. In an Intensive Care Unit, it is a fact that the professional has agility in decision making, a care free of damage, clinical knowledge and emotional control. These elements, added to life habits, conflicts and stress in the work environment can trigger disorders such as depression and anxiety in these professionals. Objective: To identify the prevalence of depression and anxiety in nursing professionals working in the adult intensive care unit. Methodology: Descriptive, cross-sectional research with quantitative approach. Because it is a census, the population was composed of a nursing team working in an Adult of two large hospitals in the public health network the municipality of Caruaru, state of the Pernambuco and that met the eligibility iteria. For data collection, a sociodemographic questionnaire and Beck's depression nd anxiety inventories were used. Descriptive statistics were adopted for data alysis. Results: The mean age of professionals was 35 years, where $86 \%$ are female most are married (46\%). It was observed that $77 \%$ of the sample did not present ressive symptoms. Regarding anxious symptoms, $85 \%$ had a minimum degree of 
anxiety. Conclusions: The results of this study showed a low prevalence of depression and anxiety. These results bring health managers the possibility of paying attention and reviewing the practices adopted in hospital institutions. It is recommended that other studies, with more robust analyses, be carried out in order to expand knowledge on the subject.

Keywords: Keywords: Depression; Anxiety; Mental health; Nursing team; Intensive Care Units

\section{RESUMEN}

Introducción: La depresión se define como un síndrome, compuesto por varios síntomas físicos y emocionales, con implicaciones para las capacidades conductuales de las personas. En una Unidad de Cuidados Intensivos, es un hecho que el profesional tiene agilidad en la toma de decisiones, una atención libre de daños, conocimiento clínico y control emocional. Estos elementos, añadidos a los hábitos de vida, conflictos y estrés en el entorno de trabajo pueden desencadenar trastornos como la depresión y la ansiedad en estos profesionales. Objetivo: Identificar la prevalencia de depresión y ansiedad en los profesionales de enfermería que trabajan en la unidad de cuidados intensivos para adultos. Metodología: Investigación descriptiva y transversal con enfoque cuantitativo. Debido a que se trata de un censo, la población estaba compuesta por un equipo de enfermería que trabajaba en una unidad adulta de dos grandes hospitales de la red de salud pública del municipio de Caruaru-Pernambuco y que cumplía con los criterios de elegibilidad. Para la recopilación de datos, se utilizó un cuestionario sociodemográfico y los inventarios de depresión y ansiedad de Beck. Se adoptaron estadísticas descriptivas para el análisis de datos. Resultados: La edad media de los profesionales fue de 35 años, donde el $86 \%$ son mujeres y la mayoría están casados (46\%). Se observó que el 77\% de la muestra no presentó síntomas depresivos. En cuanto a los síntomas ansiosos, el 85\% tenía un grado mínimo de ansiedad. Conclusiones: Los resultados de este estudio mostraron una baja prevalencia de depresión y ansiedad. Estos resultados traen a los gerentes de salud la posibilidad de prestar atención y revisar las prácticas adoptadas en las instituciones hospitalarias. Se recomienda que se lleven a cabo otros estudios, con análisis más sólidos, con el fin de ampliar el conocimiento sobre el tema.

Palabras clave: Depresión; Ansiedad; Salud mental; Equipo de enfermería; Unidades de Cuidados Intensivos. 


\section{Introdução}

A depressão é definida como uma síndrome, composta de diversos sintomas físicos e emocionais, com implicações sobre as capacidades dos indivíduos em suas esferas pessoal, social e familiar ${ }^{1}$. Suas implicações incluem alterações do sono e apetite, autoestima rebaixada, dificuldades de memória e concentração, tristeza persistente e ausência de prazer em atividades outrora prazerosas².

Dados epidemiológicos demonstram que, no Brasil, a prevalência de depressão é de 5,8\%, o equivalente a 11,5 milhões de brasileiros ${ }^{3}$. A depressão, segundo OMS, será a doença mais comum do mundo nos próximos vinte anos, sendo a doença que mais gerará custos econômicos e sociais para os governos, devido aos gastos com tratamento para a população e às perdas de produção ${ }^{3}$. Isso porque esses transtornos interferem negativamente em seu modo de viver e, principalmente, de cuidar 8 . Concernente a ansiedade, é definida como uma reação resposta ao estresse, caracterizada por sintomas como inquietação, falta de concentração, distúrbios do sono, astenia, tremores, entre outros ${ }^{4}$. A ansiedade por sua vez afeta 9,3\% (18.657.943) das pessoas que vivem no país ${ }^{3}$.

No Brasil em média 64 milhões de pessoas possuem mais de um vínculo empregatício, ultrapassando a jornada semanal prevista em lei ${ }^{5}$. Esse fenômeno é reflexo das crescentes mudanças sociais, econômicas e tecnológicas, que por conseguinte, promovem uma sociedade que trabalha 24 horas por dia, sete dias por semana $^{5}$. A equipe de enfermagem, mais especificamente, a que atua em Unidade de Terapia Intensiva (UTI), desenvolve suas atividades através de extensas jornadas de trabalho, que no geral, ocorrem em horário noturno ${ }^{5}$.

Somado a essa exaustiva carga de trabalho, há também o estresse gerado durante a realização de atividades complexas, a falta de labilidade e impotência diante o enfrentamento da morte ${ }^{6-7}$. Numa UTI, é quesito que o profissional possua agilidade tomada de decisões, um cuidado livre de danos, conhecimento e controle ocional. Esses elementos, agregados aos hábitos de vida, conflitos e estresse no biente laboral podem desencadear nesses profissionais transtornos como depressão siedade ${ }^{8-9}$. 
No que se refere a prevalência desses transtornos, a literatura é divergente. Alguns estudos evidenciaram uma baixa prevalência de depressão, sendo a ansiedade mais prevalente ${ }^{10-14}$. Entretanto outra pesquisa diverge, apontando baixa prevalência de ansiedade ${ }^{15}$. A literatura também aponta que ao se analisar a produção nacional e internacional sobre a prevalência de depressão entre os trabalhadores de enfermagem, percebe-se que o tema vem sendo menos relatado do que a ansiedade ${ }^{16}$.

Essas lacunas de conhecimento a respeito do tema, justificam a necessidade e importância da realização deste estudo, visto que se faz necessário mais pesquisas com dados consistentes afim de acrescentar novos resultados à literatura. Além de haver a necessidade de identificar a prevalência dos sintomas depressivos e ansiosos, afim de que haja devida profilaxia, tratamento e acompanhamento dos profissionais de enfermagem. Visto que um profissional em sofrimento psíquico pode causar prejuízos e danos ao paciente assistido.

\section{Metodologia}

Trata-se de uma pesquisa descritiva, transversal, com abordagem quantitativa. O estudo foi realizado em dois hospitais de grande porte da rede pública de saúde do município de Caruaru-PE. Eles foram identificados como hospital "A" e " $\mathrm{B}$ ", por questões éticas de sigilo de identidade. As UTI dessas instituições são de grande relevância para o município, visto que são referências dentro da rede de cuidados do SUS. A pesquisa foi censitária, sendo a população composta pela equipe de enfermagem que atuam em UTI Adulto.

A coleta dos dados foi realizada entre fevereiro e março de 2018. A amostra foi composta por 100 profissionais, sendo 22 enfermeiros e 78 técnicos de enfermagem. fomo critérios de elegibilidade, participaram do estudo apenas profissionais com vínculo empregatício fixo com o estabelecimento, trabalhando na UTI adulto há pelo

enos um ano, sendo exclusivo do setor e trabalhando diretamente na assistência ao ciente.

Para coleta dos dados foram utilizados instrumentos autoaplicáveis, sendo um stionário socioeconômico e demográfico, possuindo também, questões de cunho 


\section{ciência
piural}

profissional, possibilitando traçar o perfil da amostra pesquisada. Este, composto por 11 itens, possui variáveis categóricas e numéricas. Dentre as variáveis, estão: idade, gênero, estado civil, área de habitação, renda familiar, função (técnico de enfermagem ou enfermeiro), se possuíam especialização em UTI, tempo de formação, tempo de trabalho no setor, número de vínculos empregatícios e jornada semanal de trabalho.

Para verificar a prevalência de sintomas depressivos, utilizou-se o Inventário de Depressão de Beck (BDI). Criado por Aaron Beck em 1961, é composto de 21 itens, cuja intensidade varia de 0 a 3 . Para verificar a prevalência de sintomas ansiosos, o Inventário de Ansiedade de Beck (BAI). Criado por Beck e Steer em 1993, composto por 21 itens, tem por objetivo medir a gravidade dos sintomas de ansiedade. Ambos os inventários foram validados no Brasil, por Cunha no ano de 200111-12.

Os dados foram inseridos em planilha do programa Excel v2013, com dupla entrada de dados. A dupla entrada tem por objetivo reduzir a probabilidade de erros na digitação dos dados. Posteriormente, foi realizada a análise estatística descritiva, contendo medidas de tendência central e variação, além de frequências absolutas e relativas. A partir de então, os dados foram apresentados em tabelas de acordo com a normatização do periódico.

O estudo foi aprovado pelo Comitê de Ética em Pesquisa do Centro Universitário Tabosa de Almeida ASCES-UNITA, segundo número de aprovação do CEP: 2.492.744 e CAAE No 80393417.0.0000.5203. Todos os participantes assinaram o termo de consentimento livre e esclarecido, sendo-lhes assegurado o sigilo de sua identidade e a liberdade para retirar seu consentimento em qualquer fase da pesquisa.

\section{Resultados}

Dos 100 profissionais de enfermagem que compuseram a amostra do estudo, a média de idade foi de $35( \pm 8)$ anos, onde $86(86 \%)$ são do sexo feminino e sua maioria, (46\%), são casados. Quanto às características demográficas e sociais, 95 deles (95\%) ataram que moram em área urbana e apresentam renda igual ou maior que 3 lários mínimos, como pode-se observar na tabela 1. 
Tabela 1 - Distribuição das variáveis sociodemográficas dos profissionais de enfermagem que atuam na UTI Adulto de hospitais de alta complexidade do município de Caruaru-PE, Brasil, 2018.

\begin{tabular}{lll}
\hline Variável & $\mathbf{n = ( 1 0 0 )}$ & $\mathbf{( \% )}$ \\
Gênero & & \\
Feminino & 86 & 86 \\
Masculino & 14 & 14 \\
\hline Estado Civil & & \\
\hline Solteiro (a) & 45 & 45 \\
Casado (a) & 46 & 46 \\
Divorciado (a) & 3 & 3 \\
União Estável & 5 & 5 \\
Viúvo (a) & 1 & 1 \\
\hline Área de Habitação & & \\
\hline Rural & 5 & 5 \\
Urbana & 95 & 95 \\
Renda Familiar & & \\
1 salário & 4 & 4 \\
2 salários & 42 & 42 \\
3 ou mais salários & 54 & 54 \\
\hline
\end{tabular}

No que se refere às características profissionais, 78\% da amostra foi composta por técnicos de enfermagem. Dos profissionais entrevistados $(75 \%)$, não possuíam especialização para atuar em UTI. Quanto a tempo de formação, 66\% referiram ter concluído sua formação há mais de 5 anos, com média de tempo de trabalho de 43 $( \pm 44)$ meses. Sobre as condições de trabalho, os profissionais entrevistados possuem, em média, $2( \pm 1)$ vínculos empregatícios e trabalham em média $59( \pm 22)$ horas por semana, como pode-se observar na tabela 2.

Tabela 2 - Distribuição das variáveis profissionais das equipes de enfermagem que atuam na UTI Adulto de hospitais de alta complexidade do município de Caruaru-PE, Brasil, 2018.

\begin{tabular}{lcc}
\hline Variáveis & $\mathbf{n =}(\mathbf{1 0 0 )}$ & $\mathbf{( \% )}$ \\
\hline Função & & \\
\hline Técnico (a) de enfermagem & 78 & 78 \\
Enfermeiro (a) & 22 & 22 \\
\hline Possui especialização em UTI & & \\
\hline Sim & 25 & 25 \\
Não & 75 & 75 \\
\hline Tempo de Formação & & \\
\hline-2 anos & 14 & 14 \\
-4 anos & 20 & 20 \\
5 anos & 50 & 50 \\
\hline
\end{tabular}


Quando avaliado a presença de depressão e ansiedade, observou-se que 77\% da amostra não apresenta sintomas depressivos. No entanto, 22\% dos profissionais apresentam sintomas depressivo de leve a moderado. Já no que se refere aos sintomas ansiosos, 85\% apresentaram grau mínimo de ansiedade, seguido de $10 \%$ de grau leve de ansiedade, $4 \%$ de grau moderado e apenas $1 \%$ com grau severo de ansiedade.

Tabela 3 - Prevalência de sintomas depressivos e ansiosos em profissionais de enfermagem que atuam na UTI Adulto de hospitais de alta complexidade do município de Caruaru-PE, Brasil, segundo BDI e BAI*.

\begin{tabular}{lcc}
\hline Variáveis & $\mathrm{n}=(100)$ & $(\%)$ \\
\hline Score de Depressão (BDI) & & \\
\hline Não está deprimido & 77 & 22 \\
Leve a moderada & 22 & 1 \\
Moderada a severa & 1 & 0 \\
Severa & 0 & 85 \\
\hline Score de Ansiedade (BAI) & 85 & 10 \\
\hline Grau Mínimo & 10 & 4 \\
Grau Leve & 4 & 1 \\
Grau Moderado & 1 & \\
Grau Severo & & \\
*BDI: Beck Depression Inventory (Inventário de Depressão de Beck); BAI: \\
Beck. Anxiety Inventory (Inventário de Ansiedade de Beck).
\end{tabular}

\section{Discussão}

Apesar da maioria dos profissionais de enfermagem dessa pesquisa não ter apresentado sintomas depressivos, é importante ressaltar que 23 (23\%) da amostra possui sintomatologia para depressão. Assim como nos sintomas ansiosos, onde 85 (85\%) da amostra apresentaram grau mínimo de ansiedade, no entanto 15 (15\%) da população apresenta grau de ansiedade de leve a severo. Resultado semelhante foi descrito noutro estudo, cuja prevalência de depressão foi de $(28,4 \%)$, sendo $(20 \%)$ depressão leve e $(8,4 \%)$ grave (10). Concernente a ansiedade, nos profissionais de enfermagem, verificou-se prevalência de $(15 \%)$, os demais participantes (85\%) presentaram grau mínimo para ansiedade. Resultado semelhante foi encontrado na teratura onde $(15 \%)$ dos profissionais apresentaram nível de ansiedade entre leve a derada ${ }^{17}$. 
Alguns fatores podem ser predisponentes ao desenvolvimento dos transtornos depressivos e ansiosos, entre os trabalhadores de enfermagem. A literatura demonstra que fatores internos ao ambiente e processos de trabalho podem estar associados ao surgimento desses transtornos. Como exemplo desses fatores pode-se mencionar: Setores de atuação profissional, sobrecarga de serviço, relações interpessoais, assistência ao cliente, o desgaste e o suporte emocional ${ }^{18}$. Esses fatores podem ser percebidos nos resultados do estudo realizado, quando a amostra traz um maior quantitativo de técnicos de enfermagem, que majoritariamente possuem atribuições profissionais mais desgastantes com carga horária de trabalho menos flexível entre outros fatores. Isso então pode refletir de forma significativa na quantidade de profissionais com um certo grau de ansiedade e depressão. Além desses, alguns fatores externos ao trabalho também podem interferir, de acordo com a literatura, sendo eles: idade, sexo, carga de trabalho doméstico, renda familiar e estado de saúde geral do trabalhador ${ }^{18}$.

A unidade de terapia intensiva é descrita como altamente estressante e desgastante podendo estar associado ao desenvolvimento de depressão em seus trabalhadores ${ }^{10}$. Todavia, outros fatores para depressão são descritos na literatura, como idade de início precoce " $<40 "$ e sexo feminino ${ }^{19}$. Neste estudo, os profissionais possuem idade média de 35 anos $( \pm 8)$ e $86 \%$ deles são do sexo feminino, fatores que corrobora com esses achados. Relativo à ansiedade, a literatura concluiu que, profissionais de enfermagem mais novos ( $<24$ anos), apresentaram níveis mais elevados quando comparados aos mais velhos (>24 anos) ${ }^{14}$. Esse fenômeno, pode ser explicado pela falta de experiência, uma vez que, por terem iniciado a jornada laboral a menos tempo, tendem a ter maior insegurança nas ações ${ }^{14}$.

Outro fator que pode predispor à depressão é a situação conjugal. No presente estudo, o estado civil casado foi predominante (46\%). Esta variável, relativa aos rofissionais de enfermagem, quando discutida à luz da literatura, tem significância tatística no desenvolvimento de depressão ${ }^{20}$. No que se refere à ansiedade, estudo lizado em Coimbra que buscava identificar os impactos de depressão, ansiedade e nout nos profissionais de enfermagem, verificou que todos os participantes, com do civil casado, apresentaram um nível moderado de ansiedade ${ }^{14}$. $\mathrm{O}$ acúmulo de 
papéis, dentre eles, o familiar, pode acarretar menos tempo para atividades de lazer e descanso, fatores que contribuem para o aumento de suas incidências ${ }^{20}$.

Outro fator que pode desencadear a depressão é a renda familiar ${ }^{21}$. Quanto menor a remuneração, maior a prevalência de depressão ${ }^{22}$. A renda dos profissionais inquiridos neste estudo foi de 3 ou mais salários mínimos (54\%). Contudo, cabe ressaltar que, esse montante, é o resultado final da soma dos vínculos profissionais. A média de vínculos empregatícios neste trabalho foi de $2( \pm 1)$. Resultado semelhante foi descrito em outra pesquisa realizada em três instituições hospitalares de uma cidade do noroeste do Estado São Paulo, cuja maior parte da amostra também possuía mais de um vínculo empregatício ${ }^{10}$. A dupla jornada de trabalho é uma alternativa, para que os profissionais possuam, mesmo diante de baixa remuneração, melhores salários ${ }^{10}$.

Mesmo sendo comum, a dupla jornada de trabalho pode interferir em alguns aspectos referentes à qualidade de vida do trabalhador, sendo considerado pela literatura como um fator de associação com a depressão ${ }^{10}$. Relativo a ansiedade, pesquisa realizada com os profissionais de enfermagem em uma Unidade de Terapia Intensiva de um Hospital do Interior de Santa Catarina, identificou no discurso dos entrevistados que a renda e quantidade de vínculos empregatícios influenciam, também, o surgimento da ansiedade ${ }^{12}$. Dessa forma, profissionais que praticam dupla jornada de trabalho, são mais estressados em relação àqueles que possuem jornada única, sendo o estresse, consequentemente, um fator determinante para a ansiedade ${ }^{23}$. Isso pode ser percebido nos resultados deste estudo, que retratam a maior parte dos profissionais entrevistados, levando em conta que os profissionais de enfermagem no geral atualmente não possuem um piso salarial, possui a renda familiar de 3 ou mais salários mínimos e que a maior parte deles também possui estado civil solteiro, ou seja, reflete implicitamente que estes profissionais podem ter mais de um vínculo empregatício, acumulando funções e trazendo maior desgaste físico e emocional, hdo um fator predisponente tanto de ansiedade como de depressão.

Quanto à categoria profissional, 78\% da amostra constituiu-se por técnicos em fermagem. Um estudo realizado em Santa Catarina apresentou valores semelhantes 
onde $76,93 \%$ eram técnicos de enfermagem ${ }^{12}$. A literatura afirma que técnicos e auxiliares de enfermagem carecem de um controle emocional maior, pois ao lidarem com o sofrimento em seu dia-a-dia, possuem maior risco de desenvolver sofrimento psíquico ${ }^{24}$. A categoria de técnicos em enfermagem é maior que a de enfermeiros e isso se explica pela própria divisão do trabalho, sendo esses profissionais responsáveis pela maioria dos procedimentos técnicos ${ }^{25}$. Além disso, as relações hierárquicas e de poder existentes intra e inter equipes contribuem para um maior desgaste emocional dessa categoria2 $2^{5}$.

Apesar da função ter sido apontada como risco emocional, esperava-se que outras variáveis como especialização, tempo de formação e tempo de trabalho na UTI, também fossem fatores que influenciariam na manifestação da depressão e ansiedade como exposto nos resultados deste estudo que mostram a maioria, profissionais com mais de 5 anos de profissão e que não possuem especialização em UTI. Entretanto, a literatura é discrepante quanto a isso. A mesma aponta que não houve associação entre estes transtornos e as variáveis: especialização, tempo de formação e tempo de trabalho no setor ${ }^{10}$.

Relativo à jornada semanal de trabalho, a média de horas trabalhadas foi de 59 $( \pm 22)$ horas. Tal jornada, além de exaustiva, ultrapassa o que é previsto em lei. A literatura aponta a carga de trabalho, como um dos fatores estressores mais consistentes e significantes, ou seja, quanto maior a sobrecarga de trabalho, mais casos de depressão são observados entre os profissionais de enfermagem ${ }^{25}$. No que condiz à ansiedade, o número de horas trabalhadas, é um fator que influencia na tensão e na ansiedade dos profissionais ${ }^{25}$. Um estudo realizado na china, com 2.641 médicos que trabalhavam em hospitais públicos em Shenzhen, constatou que a rotina de trabalho com carga horária maior que 60 horas semanais possui relação com altos níveis de ansiedade ${ }^{15}$. Outros fatores que podem influenciar nos níveis de ansiedade são, a instabilidade dos pacientes e a sobrecarga de trabalho ${ }^{25}$.

Referente aos resultados do estudo, supõe-se que os profissionais podem ter tado por omitir algumas informações, por terem medo de expressar seus timentos ou não sentir-se a vontade para relatar suas angústias, levando a tal 


\section{ciência
piural}

resultado da pesquisa. Relacionado às limitações do estudo menciona-se o fato de não ser possível verificar quais eram os fatores associados à depressão e ansiedade nos profissionais, visto que a análise descritiva não permite esse tipo de abordagem. Outra limitação, ocorreu durante a coleta de dados, devido disponibilidade por parte dos profissionais em responderem os instrumentos de coleta. Isso ocorreu pela demanda de atividades que os mesmos realizam na UTI, dificultando assim que os profissionais respondessem aos instrumentos com tranquilidade.

\section{Conclusões}

A qualidade da assistência prestada ao cliente é multifatorial e possui relação direta com à saúde mental dos profissionais de enfermagem. Os resultados desse estudo evidenciaram uma baixa prevalência de depressão e ansiedade. Contudo, cuidado especial deve ser destinado aos profissionais que apresentaram algum grau destes transtornos, pois, as variáveis aqui discutidas, podem culminar em perda de produção, levando à prejuízos na qualidade da assistência.

Apesar das limitações apontadas, este estudo torna-se relevante, tendo em vista que foi pioneiro ao verificar a prevalência de tais transtornos nas UTI's públicas do município de Caruaru, referência no interior do estado. Além disso, tais resultados trazem aos gestores de saúde a possibilidade de atentar-se e rever as práticas adotadas nas instituições hospitalares que podem estar influenciando na saúde psíquica de seus colaboradores. O que lhes permite buscar ações que busque ofertar ações de apoio aos profissionais, principalmente aos que apresentaram algum grau dos transtornos depressivos e ansiosos, possibilitando uma melhor qualidade de vida no ambiente laboral, visando a minimização de tais transtornos.

Recomenda-se que outros estudos, com análises mais robustas, sejam realizados no intuito de se ampliar o conhecimento sobre a temática. Propõe-se, ainda, que tais estudos sejam correlacionais com as atividades desenvolvidas no setor e/ou com atores externos ao ambiente da UTI. Visto que não se pode afirmar que os ofissionais estejam deprimidos ou ansiosos apenas pelas suas atividades laborais na , pois os fatores externos também podem influenciar nas suas emoções. 


\section{Referências}

1. Etapechusk J, Fernandes LRS. Depressão sob o olhar gestáltico. Psicologia.pt [Internet]. 2018 Fev [cited 2018 05/05]. Disponível em: http://www.psicologia.pt/artigos/textos/A1171.pdf

2. American Psychiatric Association. DSM-5: Manual Diagnóstico e Estatístico de Transtornos Mentais. 5a ed. Porto Alegre: Artmed; 2014.

3. PAHO. Determinantes sociais e riscos para a saúde, doenças crônicas não transmissíveis e saúde mental. [internet] [cited 2018 03/10]. Disponível em: http:/ $/$ www.paho.org/bra/index.php?option $=$ com_content\&view $=$ article\&id=5354: aumenta-o-numero-de-pessoas-com-depressao-no-mundo\&Itemid $=839$

4. Costa KMV, Sousa KRS, Formiga PA, Silva WS, Bezerra EBN. Ansiedade em universitários na área da saúde. In: Anais do 2. Congresso Brasileiro de Ciências da Saúde; 2017 Jun 14-16; Campina Grande, Brasil. Campina Grande: Faculdade Maurício de Nassau; 2017. p. 1-10.

5. Moreno CRC, Fischer FM, Rotenberg L. A saúde do trabalhador na sociedade 24 horas. São Paulo em perspectiva [internet]. 2003 Jan/Mar [cited 2018 03/08]; 17 (1): 3436. Disponível em: http://dx.doi.org/10.1590/S0102-88392003000100005

6. Gomes GC, Lunardi Filho WD, Erdmann AL. O Sofrimento Psíquico em Trabalhadores de UTI Interferindo no seu Modo de Viver a Enfermagem. R Enferm UERJ [internet]. 2006 Jan/Mar [cited 2018 03/05]; 14 (1): 93-9. Disponível em: http://repositorio.furg.br/handle/1/1541

7. Alves EF. Cuidador de Enfermagem e o Cuidar em Uma Unidade de Terapia Intensiva. UNOPAR Cient Ciênc Biol Saúde [internet]. 2012 Mar [cited 2018 03/28]; 15 (2): 115-22. Disponível em:

https://www.researchgate.net/publication/235971663_O_cuidador_de_enfermage m_e_o_cuidar_em_uma_Unidade_de_Terapia_Intensiva_The_Nursing_Caregiver_a nd_the_Caring_in_Intensive_Care_Units

8. Afecto MCP, Teixeira MB. Avaliação do estresse e da síndrome de burnout em enfermeiros que atuam em uma unidade de terapia intensiva: Um estudo qualitativo. Obj Nursing [internet]. 2009 [cited 2018 03/08]; 8 (1): 1-12. Disponível em: http://www.objnursing.uff.br/index.php/nursing/rt/printerFriendly/j.16764285.2 $009.2107 / 453$

Martins JT, Ribeiro RP, Remijo KP, Ribeiro PHV. Mental disorders related to work nursing: integrative review. JNurs UFPE on line [Internet]. 2014 Jun [cited 2018 27]; 8 (6): 1746-56. Disponível em:

ps:// periodicos.ufpe.br/revistas/revistaenfermagem/article/view/13650/16516 
10. Vargas D, Dias APV. Prevalência de depressão em trabalhadores de enfermagem de Unidade de Terapia Intensiva: estudo em hospitais de uma cidade do noroeste do Estado São Paulo. Rev. Latino-Am. Enfermagem [internet]. 2011 set/out. [cited 2018 03/22]; 19 (5): [09 telas]. Disponível em:

http://www.scielo.br/pdf/rlae/v19n5/pt_08.pdf

11. Barbosa KKS, Vieira KFL, Alves ERP, Virgínio NA. Sintomas depressivos e ideação suicida em enfermeiros e médicos da assistência hospitalar. Rev Enferm UFSM [Internet]. 2012. [cited 2018 05/07]; 2 (3): 515-522. Disponível em: https:// periodicos.ufsm.br/reufsm/article/view/5910/pdf

12. Batista FCN, Pawlowytsch PWM. Aspectos emocionais de depressão, ansiedade, desesperança e ideação suicida nos profissionais da unidade de terapia intensiva de um hospital do interior de Santa Catarina. Saúde Meio Ambient [internet]. 2012 jun. [cited 2018 05/09]; 1 (1): 188-202. Disponível em: http://www.periodicos.unc.br/index.php/sma/article/viewFile/228/271

13. Machado DA, Figueiredo NMA, Velasques LS, Bento CAM, Machado WCA, Vianna LAM. Cognitive changes in nurses working in intensive care units. Rev Bras Enferm [Internet]. 2018 [cited 28/03]; 71 (1): 73-79. Disponível em: http://www.scielo.br/pdf/reben/v71n1/0034-7167-reben-71-01-0073.pdf

14. Oliveira V, Pereira T. Ansiedade, depressão e burnout em enfermeiros - Impacto do trabalho por turnos. Revista de Enfermagem Referência [internet]. 2012 jul. [cited 2018 05/07]; III Série - 7: 43-54. Disponível em: http:/ / www.scielo.mec.pt/pdf/ref/vserIIIn7/serIIIn7a05.pdf

15. Gong Y, Han T, Chen W, Dib HH, Yang G, Zhuang R, et al. Prevalence of anxiety and depressive symptoms and related risk factors among physicians in China: a crosssectional study. PLOS ONE [Internet]. 2014 Jul [cited 2018 05/08]; 9 (7): 103-242. Disponível em:

http://journals.plos.org/plosone/article/file?id=10.1371/journal.pone.0103242\&typ $\mathrm{e}=$ printable

16. Franco GP, Barros ALBL, Nogueira-Martins LA. Qualidade vida e sintomas depressivos em residentes de enfermagem. Rev Latino-am Enfermagem [internet]. 2005 Mar/Abr [cited 2018 05/18]; 13 (2): 139-44. Disponível em: http:/ / www.scielo.br/scielo.php?script=sci_arttext\&pid=S0104-11692005000200002

17. Kaplan H, Sadock BJ, Grebb JA. Compêndio de Psiquiatria: Ciências do comportamento e Psiquiatria clínica. 7. ed. Porto Alegre: Artmed; 1997.

Manetti ML, Marziale MHP. Fatores associados à depressão relacionada ao abalho de enfermagem. Estud Psicol. [internet]. 2007 [cited 2018 05/18]; 12 (1): 79-85. sponível em: http://www.scielo.br/scielo.php?script=sci_arttext\&pid=S14134X2007000100010 
19. Patterson J, Albala AA, Mccahill ME, Edwards TM. Guia de Psicofarmacologia para o terapeuta: Trabalhando com pacientes, suas famílias e seus médicos para aperfeiçoar o tratamento. 1. ed. São Paulo - SP: Roca; 2010.

20. Chiang YM, Chang Y. Stress, depression, and intention to leave among nurses in different medical units: Implications for healthcare management/nursing practice. Health Policy [internet]. 2012. [cited 2018 05/06]; 108 (2-3): 149-57. Disponível em: https://www.healthpolicyjrnl.com/article/S0168-8510(12)00252-7/fulltext

21. Freimann T, Merisalu E. Work-related psychosocial risk factors and mental health problems amongst nurses at a university hospital in Estonia: a cross-sectional study. Scand J Public Health [internet]. 2015 [cited 2018 05/07]; 43 (5): 447-452. Disponível em: http://journals.sagepub.com/doi/pdf/10.1177/1403494815579477

22. Vieira TG, Beck CLC, Dissen CM, Camponogara S, Gobatto M, Coelho APF. Adoecimento e uso de medicamentos psicoativos entre trabalhadores de enfermagem de unidades de terapia intensiva. Rev Enferm UFSM [Internet]. 2013 [cited 2018 05/08]; 3 (2): 205-14. Disponível em: http://cascavel.ufsm.br/revistas/ojs2.2.2/index.php/reufsm/article/view/7538

23. Santos FD, Cunha MHF, Robazzi MLCC, Pedrão LJ, Silva LA, Terra FS. O estresse do enfermeiro nas unidades de terapia intensiva adulto: uma revisão da literatura. SMAD, Rev Eletrônica Saúde Mental Álcool Drog [Internet]. 2010 [cited 2018 05/06]; 6 (1): 1-16. Disponível em: http:/ / pepsic.bvsalud.org/pdf/smad/v6n1/14.pdf

24. Gomes RK, Oliveira VB. Depressão, ansiedade e suporte social em profissionais de enfermagem. Boletim de Psicologia [Internet]. 2013 [cited 2018 05/06]; 63 (138): 23-33. Disponível em: http://pepsic.bvsalud.org/pdf/bolpsi/v63n138/v63n138a04.pdf

25. Veloso LUP, Laurinho LMB, Sousa LRP, Veloso CSJ, Monteiro FJG, Souza CF. Prevalence of anxiety in nursing professionals of urgency and emergency. J Nurs UFPE On line [Internet]. 2016 Nov [cited 2018 05/07]; 10 (11): 3969-76. Disponível em: https://periodicos.ufpe.br/revistas/revistaenfermagem/article/view/11479/13328 